\title{
A Note on Inverse Moments of Binomial Variates
}

\author{
Francisco Cribari-Neto*
}

Nancy Lopes Garcia**

Klaus L. P. Vasconcellos***

\section{Abstract}

We show that the inverse moments of a binomial random variable are such that

$$
\mathbb{E}\left[(1+X)^{-\alpha}\right]=O\left((n p)^{-\alpha}\right) \quad \text { as } n \rightarrow \infty
$$

for all $\alpha \in \mathbb{R}$.

\section{Resumo}

Mostramos que os momentos inversos de uma variável aleatória binomial são tais que

$$
\mathbb{E}\left[(1+X)^{-\alpha}\right]=O\left((n p)^{-\alpha}\right) \text { quando } n \rightarrow \infty
$$

para todo $\alpha \in \mathbb{R}$.

Key Words: inverse moments, binomial random variable, moment generating function.

JEL Code: C10; C13.

"Departamento de Estatística/CCEN, Universidade Federal de Pernambuco, Recife/PE 50740540, Brazil; e-mail: cribari@npd.ufpe.br

** Departamento de Estatística/IMECC, Universidade Estadual de Campinas, Caixa Postal 6065, Campinas/SP 13081-970, Brazil; e-mail: nancy@ime.unicamp.br

***Departamento de Estatística/CCEN, Universidade Federal de Pernambuco, Recife/PE 50740540, Brazil; e-mail: klaus@de.ufpe.br

Brazilian Review of Econometrics Rio de Janeiro v.20, $\mathrm{n}$ 을, pp.269-277 Nov.2000 
A Note on Inverse Moments of Binomial Variates

\section{Introduction.}

Oftentimes in econometrics, statistics and probability one needs to evaluate inverse moments of random variables. For instance, Bock, Judge and Yancey (1984) and Xie (1988) obtain expressions for inverse moments of non-central chi-squared variates. In this note we address the issue of binomial inverse moments evaluation.

The binomial distribution was derived by James Bernoulli in 1713 for the case where the probability of success $p$ equals $r /(r+s)$, where $r$ and $s$ are positive integers, and had been previously considered by Pascal for the case where $p=1 / 2$. McKendrick (1914) obtained the binomial distribution as the solution to the birthand-emigration process, and Haight (1957) has shown that the distribution arises in the context of the $M / M / 1$ queue with balking, provided that the arrival rate of customers in the queue is $(N-n) /[N(n+1)]$, where there are $n$ customers and $N$ denotes the maximum queue size. The binomial distribution can also be viewed as the stationary distribution for the Ehrenfest model. It has a number of applications on many different fields. For instance, in genetics it arises in the study of the number of nucleotides that are in the same state in two DNA sequences (Kaplan and Risko, 1982). The binomial distribution has also applications in ecology (e.g., Seber, 1982), mortality data (e.g., Seal, 1949), etc. It should also be remarked that the number of defective items in random samples of size $n$ from a stable production process follows a binomial distribution. For that, it has a number of applications in the area of quality control. The binomial distribution is also used for distribution-free inference on distribution values, distribution quantiles, and tolerance limits for distributions. For further details on this distribution and its applications, see Bury (1999, Chapter 6) and Johnson, Kotz and Kemp (1993, Chapter 3).

Inverse moments for binomial random variables appear in several 
Francisco Cribari-Neto, Nancy Lopes Garcia \& Klaus L. P. Vasconcellos contexts. The evaluation of moments of the form

$$
\mathbb{E}\left[\frac{1}{(1+X)^{\alpha}}\right]
$$

is necessary for bounds used in asymptotic results. For example, in Garcia and Palacios (2000) bounds for the mixing time of a random walk are estimated in terms of the above moments for $p=1 / 2$. As early as Stephan (1945) and as recent as Marciniak and Wesolowski (1999), there has been interest in finding closed-form expressions, approximations or bounds for inverse moments, especially for the binomial distribution with view toward applications in life testing, Bayesian post-stratification and Stein estimators among others. In particular, when $\alpha$ is a non-negative integer it is possible to use a strategy described in Chao and Strawderman (1972) and Cressie et al. (1981). It consists of successively integrating the moment generating function $M_{X}(t)$ of $X$, namely:

$$
\mathbb{E}\left[\frac{1}{(1+X)^{k}}\right]=\int_{0}^{\infty} \int_{t_{1}}^{\infty} \cdots \int_{t_{k-1}}^{\infty} M_{X}\left(-t_{k}\right) e^{-t_{k}} d t_{k} \cdots d t_{2} d t_{1}
$$

We either make the changes of variables $e^{-t_{i}}=s_{i}, 1 \leq i \leq k$, or exchange the order of integration in (2) to obtain the desired expressions. However, even in the binomial case, where $M_{X}(t)=$ $\left(1-p+p e^{t}\right)^{n}$, there are no closed-form expressions except when $\alpha=1$, as was observed in Chao and Strawderman (1972), in which case we get

$$
\mathbb{E}\left[\frac{1}{(1+X)}\right]=\frac{1}{p(n+1)}\left[1-(1-p)^{n+1}\right]
$$

Nevertheless, there are situations in which the exact expression is not important and where asymptotic results can be useful. Lower 
bounds can be obtained easily by Jensen inequality. Bounds have been studied extensively in several cases. Wooff (1985) and Pittenger (1990) develop different distribution-free methods and get the same upper bound when $\alpha$ is a positive integer. However, for large $n$ they do not achieve the right order of magnitude $O\left((n p)^{-\alpha}\right)$. One important information is to get the order of the expression in (1) and the leading constant. Garcia and Palacios (2001) present a proof for $\alpha>0$ based on a normal approximation. We offer an alternative proof for $\alpha>0$ based on the moment generating function, and argue that the result also holds for $\alpha<0$.

\section{Approximating $\mathbb{E}\left[\frac{1}{(1+X)^{\alpha}}\right]$}

Consider the function $f(x)=(1+x)^{-\alpha}$. Our interest lies in $\mathbb{E}[f(X)]$ where $X \sim \operatorname{Bin}(n, p), n \in \mathbb{N}$ and $0<p<1$, with $n$ assumed large. At the outset, let $\alpha>0$. We write

$$
f(x)=\frac{1}{\Gamma(\alpha)} \int_{0}^{\infty} e^{-(1+x) t} t^{\alpha-1} d t
$$

where $\Gamma(\cdot)$ is the gamma function. Thus,

$$
\begin{aligned}
\mathbb{E}[f(X)] & =\frac{1}{\Gamma(\alpha)} \int_{0}^{\infty} t^{\alpha-1} e^{-t} \mathbb{E}\left[e^{-t X}\right] d t \\
& =\frac{1}{\Gamma(\alpha)} \int_{0}^{\infty} t^{\alpha-1} e^{-t}\left(1-p+p e^{-t}\right)^{n} d t
\end{aligned}
$$

where the last equality follows from the moment generating function for the binomial distribution. Now use the change of variable (1$\left.p+p e^{-t}\right)=e^{-r}$. Thus $t=-\log \left[p^{-1}\left(e^{-r}+p-1\right)\right]$ and $e^{-t} d t=$ $p^{-1} e^{-r} d r$. Therefore, 
Francisco Cribari-Neto, Nancy Lopes Garcia \& Klaus L. P. Vasconcellos

$$
\mathbb{E}[f(X)]=\int_{0}^{-\log (1-p)} g(r) e^{-r n} d r
$$

where

$$
g(r)=p^{-1} e^{-r} \frac{1}{\Gamma(\alpha)}\left\{-\log \left[p^{-1}\left(e^{-r}+p-1\right)\right]\right\}^{\alpha-1} .
$$

We have the following series expansion for $g(r)$ :

$$
g(r)=p^{-\alpha} r^{\alpha-1} \frac{1}{\Gamma(\alpha)}\left[1+\frac{1}{2}\left(\frac{\alpha-1}{p}-(\alpha+1)\right) r+O\left(r^{2}\right)\right]
$$

Expansion (3) was obtained from the following expressions: $\log (1+$ $z)=z-z^{2} / 2+O\left(z^{3}\right)$ for $|z|<1, e^{-z}=1-z+O\left(z^{2}\right)$, and $(1+z)^{\nu}=$ $1+\nu z+O\left(z^{2}\right)$ for $z>-1$.

Fix any $0<\delta<\min \{1,-\log (1-p)\}$. For $0<r<\delta$,

$$
\int_{0}^{\delta} r^{j} e^{-r n} d r=\frac{\Gamma(j+1)}{n^{j+1}}+O\left(e^{-\delta n}\right)
$$

Equation (4) can be established as follows:

$$
\begin{aligned}
\int_{0}^{\delta} r^{j} e^{-r n} d r & =\int_{0}^{\infty} r^{j} e^{-r n} d r-\int_{\delta}^{\infty} r^{j} e^{-r n} d r \\
& =\frac{\Gamma(j+1)}{n^{j+1}}-\frac{\Gamma(j+1, \delta n)}{n^{j+1}}
\end{aligned}
$$

where $\Gamma(\cdot, \cdot)$ denotes the incomplete gamma function. Now, expand $\Gamma(j+1, \delta n)$ as (see Abramowitz and Stegun 1965, §6.5.32, p. 263) 
A Note on Inverse Moments of Binomial Variates

$$
\Gamma(j+1, \delta n)=(\delta n)^{j} e^{-\delta n}\left[1+\frac{j}{\delta n}+\frac{j(j-1)}{\delta^{2} n^{2}}+\cdots\right]
$$

and the result follows. Moreover,

$$
\int_{\delta}^{-\log (1-p)} g(r) e^{-r n} d r=O\left(e^{-\delta n}\right)
$$

Noticing that the remaining terms in (4) and (5) go to 0 exponentially fast, we have

$$
\begin{aligned}
\mathbb{E}[f(X)] & =\int_{0}^{\delta} g(r) e^{-r n} d r+\int_{\delta}^{-\log (1-p)} g(r) e^{-r n} d r \\
& =\int_{0}^{\delta} p^{-\alpha} r^{\alpha-1} \frac{1}{\Gamma(\alpha)}\left[1+\frac{\tau}{2}\left(\frac{\alpha-1}{p}-(\alpha+1)\right)+\cdots\right] e^{-r n} d r+O\left(e^{-\delta n}\right) \\
& =\frac{1}{\Gamma(\alpha)} p^{-\alpha}\left[\frac{\Gamma(\alpha)}{n^{\alpha}}+\left(\frac{\alpha-1}{2 p}-\frac{\alpha+1}{2}\right) \frac{\Gamma(\alpha+1)}{n^{\alpha+1}}\right]+O\left(n^{-(\alpha+2)}\right) \\
& =(n p)^{-\alpha}+O\left(n^{-(\alpha+1)}\right) .
\end{aligned}
$$

Notice now that the upper bound given by Wooff (1985) and Pittenger (1990) is

$$
\frac{(1-p)}{(1-p)+n p}+\frac{n p}{(1-p)+n p}(1+n p+(1-p))^{-\alpha}
$$

which is greater than the previous equation for large $n$.

Also, not only do we have the leading term $n^{-\alpha}$, but also higher order terms. For example, the term of order $n^{-(\alpha+1)}$ is

$$
\begin{aligned}
E\left[(1+X)^{-\alpha}\right]= & (n p)^{-\alpha}+\left(\frac{\alpha-1}{2 p}-\frac{\alpha+1}{2}\right) \frac{\Gamma(\alpha+1)}{\Gamma(\alpha)} \frac{1}{n^{\alpha+1} p^{\alpha}} \\
& +O\left(n^{-(\alpha+2)}\right) .
\end{aligned}
$$


Now let $\alpha<0$ and $\gamma=-\alpha$ We have that $(1+X)$ /n-bas the same distribution as $1 / n+\bar{Y}$, where $\bar{Y}=n^{-1} \sum_{i=1}^{n} Y_{i}$ with $Y_{1}, \ldots, Y_{n}$ being a sequence of independent Bernoulli trials with probability of success equal to $p$. But

$$
\left(\frac{1}{n}+\bar{Y}\right)^{\gamma} \leq 2^{\gamma}
$$

since $0<n^{-1}+\bar{Y} \leq 2$. We also know by the Strong Law of Large Numbers that $\bar{Y} \stackrel{\text { a.s.s. }}{\longrightarrow} p$. Therefore, by the Bounded Convergence Theorem,

$$
\mathbb{E}\left(\frac{1}{n}+\bar{Y}\right)^{\gamma} \rightarrow p^{\gamma}
$$

More specifically,

$$
\left|\mathbb{E}\left(\frac{1}{n}+\bar{Y}\right)^{\gamma}-p^{\gamma}\right|=o(1)
$$

as $n \rightarrow \infty$.

Equivalently,

$$
\mathbb{E}\left[\frac{1}{(1+X)^{\alpha}}\right]=O\left((n p)^{-\alpha}\right)+o\left(n^{-\alpha}\right)
$$

as $n \rightarrow \infty$.

The result thus holds for all $\alpha \in \mathbb{R}$.

Acknowledgments.

We thank two anonymous referees for comments and suggestions. We also gratefully acknowledge financial support from $\mathrm{CNPq}$ and FAPESP. 
Submitted in February 2001. Revised in October 2001.

\section{References}

Abramowitz, M. \& I. A. Stegun 1965. Handbook of Mathematical Functions with Formulas, Graphs and Mathematical Tables. New York: Dover.

Bock, M. E., G. G. Judge, \& T. A. Yancey 1984. "A simple form for the inverse moments of non-central $\chi^{2}$ and $F$-random variables and certain confluent hypergeometric-functions". Journal of Econometrics, 25:217-234.

Bury, K. 1999. Statistical Distributions in Engineering. New York: Cambridge University Press.

Chao, M. T. \& W. E. Strawderman 1972. "Negative moments of positive random variables". Journal of the American Statistical Society, 67:429-431.

Cressie, N., A. S. Davis, J. L. Folks, \& G. E. Policello, 1981. "The moment-generating function and negative integer moments". The American Statistician, 35:148-150.

Garcia, N. L. \& J. L. Palacios 2000. "On mixing times for stratified walks on the $d$-cube". Preprint in Los Alamos Archives. http://arXiv.org/abs/physics/0003006.

Garcia, N. L. \& J. L. Palacios 2001. "On inverse moments of nonnegative random variables". Statistics and Probability Letters, 53:235-239.

Haight, F. A. 1957. "Queueing with balking". Biometrika, 44:360369.

Johnson, N. L., S. Kotz, \& A. W. Kemp, 1993. Univariate Discrete Distributions, 2nd ed. New York: Wiley.

Kaplan, N. \& K. Risko 1982. "A method for estimating rates of 
nucleotide substitution using DNA sequence data". Theoretical Population Biology, 21:318-328.

Marciniak, E. \& J. Wesołowski 1999. "Asymptotic Eulerian expansions for binomial and negative binomial reciprocals". Proceedings of the American Mathematical Society, 127:3329-3338.

McKendrick, A. G. 1914. "Studies on the theory of continuous probabilities, with special reference to its bearing on natural phenomena of a progressive nature". Proceedings of the London Mathematical Society, 13:401-416.

Pittenger, A. O. 1990. "Sharp mean-variance bounds for Jensentype inequalities". Statistics and Probability Letters, 10:91-94.

Seal, H. L. 1949. "Mortality data and the binomial probability law". Skandinavisk Aktuarietidskrift, 30:18-43.

Seber, G. A. F. 1982. The Estimation of Animal Abundance, 2nd ed. London: Griffin.

Stephan, F. F. 1945. "The expected value and variance of the reciprocal and other negative powers of a positive Bernoullian variate". Annals of Mathematical Statistics, 16: 50-61.

Wooff, D. A. 1985. "Bounds on reciprocal moments with applications and developments in Stein estimation and poststratification". Journal of the Royal Statistical Society B, 47:362-371.

Xie, W. Z. 1988. "A simple way of computing the inverse moments of a non-central chi-square random variable". Journal of Econometrics, 37:389-393. 


$$
\text { . }
$$

